\title{
Inmunoterapia activa en cáncer de próstata: Revisión con atención especial a las células dendríticas
}

\author{
Thomas-Kaskel AK, Veelken H. \\ Departamento de Medicina Interna I (Hematología/Oncología) y Comprehensive Cancer Center, \\ Freiburg University Medical Center, Freiburg, Alemania.
}

Actas Urol Esp. 2007;31(6):668-679

\section{RESUMEN}

\section{INMUNOTERAPIA ACTIVA EN CÁNCER DE PRÓSTATA: REVISIÓN CON ATENCIÓN ESPECIAL A} LAS CÉLULAS DENDRÍTICAS

El cáncer de próstata recurrente o metastásico generalmente se considera incurable.

Dado que el beneficio del tratamiento de deprivación hormonal es transitorio y los éxitos de la quimioterapia sistémica limitados, son necesarias modalidades alternativas de tratamiento, tanto para la recurrencia de PSA como para la enfermedad hormono-refractaria. Las células cancerígenas prostáticas expresan varios antígenos asociados a tumores que están siendo evaluados actualmente como dianas para abordajes de inmunoterapia activa y específica. Las células dendríticas son las células presentadoras de antígenos más potentes, capaces de activar las células T vírgenes y romper la tolerancia periférica, y así inducir respuestas inmunes a tumores. Hasta la fecha, cerca de 1.000 pacientes con cáncer de próstata han sido tratados con inmunoterapia activa, basada en células dendríticas o de otro tipo. Se han comunicado respuestas inmunes inducidas por la vacunación en dos tercios de los ensayos clínicos con células dendríticas, y cambios favorables de la evolución clínica de la enfermedad en casi la mitad de los pacientes tratados. Sin embargo, la mayoría de las respuestas fueron modestas y transitorias. Por lo tanto, los mecanismos de fallo del tratamiento y las posibilidades de mejoría de la eficacia de la vacunación están siendo discutidos.

Palabras clave: Inmunoterapia. Cáncer de próstata. Células dendríticas.

\section{ABSTRACT}

\section{ACTIVE IMMUNOTHERAPY OF PROSTATE CANCER WITH A FOCUS ON DENDRITIC CELLS}

Recurrent or metastatic prostate cancer is generally considered an incurable disease. Given the transient benefit from hormone deprivation therapy and limited successes of systemic chemotherapy, alternative treatment modalities are needed both in the situation of PSA recurrence and in hormonerefractory disease. Prostate cancer cells express several tumor associated antigens which are currently being evaluated as targets for active and specific immunotherapy approaches. Dendritic cells (DC) are the most powerful antigen-presenting cells (APC), able to prime naïve T cells and to break peripheral tolerance and thus induce tumor immune responses. Close to 1000 prostate cancer patients have been treated with DC-based or other forms of active immunotherapy to date. Vaccination-induced immune responses have been reported in two thirds of DC trials, and favorable changes in the clinical course of the disease in almost half of the patients treated. Most responses, however, were modest and transient. Therefore, mechanisms of treatment failure and possibilities to improve vaccination efficacy are being discussed. 
$\mathrm{E}^{1}$ cáncer de próstata representa la segunda causa principal de mortalidad por cáncer en varones en los Estados Unidos de América y la tercera causa en Europa ${ }^{1,2}$. La cirugía y la radioterapia son opciones de tratamiento curativas en la enfermedad localizada ${ }^{3}$. Sin embargo, hasta el $40 \%$ de los pacientes experimenta un aumento del PSA después del tratamiento primario de intención curativa, y un tercio de estas recurrencias de PSA desarrollan metástasis demostrables. La mediana de tiempo desde la detección de la primera metástasis hasta la muerte es algo menor de 5 años $^{4}$. En la enfermedad hormonodependiente, el tratamiento paliativo de deprivación androgénica puede inducir remisiones hasta en el $80 \%$ de los pacientes, pero después de 15 a 20 meses, con regularidad las células tumorales se hacen andrógeno independientes ${ }^{5}$. En esta situación, la quimioterapia con docetaxel induce respuestas en cerca de la mitad de los pacientes y ofrece un beneficio significativo en supervivencia. Sin embargo, la mediana de tiempo hasta la progresión es sólo de 6-7 meses y la mediana de supervivencia global 18,9 meses $^{6,7}$. Por lo tanto, se requieren urgentemente nuevas estrategias de tratamiento alternativo.

\section{INMUNOLOGÍA TUMORAL DEL CÁNCER DE PRÓSTATA}

Las células de cáncer de próstata expresan varias proteínas restrictivas de tejido que pueden servir como antígenos asociados a tumor (AAT) para la inmunoterapia activa. Los ensayos clínicos de inmunización generalmente buscan inducir respuestas inmunes específicas contra uno o más de éstos antígenos. Un prerrequisito crucial para el reconocimiento inmune es la presentación de los péptidos derivados de AAT dentro del complejo HLA de las células cancerígenas a células $\mathrm{T}$ que reconocen esta estructura por medio de un receptor de células $\mathrm{T}$ específico.

$\mathrm{El}$ antígeno prostático específico (PSA) es una proteasa sérica de $34 \mathrm{kD}$ expresada exclusivamente en las células epiteliales prostáticas y virtualmente en todos los cánceres de próstata adenomatosos ${ }^{8}$. El PSA circula de sangre periférica debido a la pérdida desde la superficie celular. Además de su utilización diagnóstica como marcador tumoral sérico, el PSA es el antígeno más ampliamente utilizado en los ensayos clínicos de inmunoterapia. Se ha descrito que varios péptidos derivados del PSA son presentados por el HLA-A2 e inducen linfocitos T citotóxicos específicos in vitro ${ }^{9-11}$.

$\mathrm{El}$ antígeno prostático específico de membrana (PSMA) es una glicoproteína de membrana tipo II expresada en células epiteliales prostáticas normales y malignas, pero también en algunos otros órganos ${ }^{12}$. Se han identificado varios péptidos PSMA restringidos por HLA-A2.

La fosfatasa ácida prostática (FAP) es una glicoproteína con actividad enzimática presente en el semen humano. La expresan las células epiteliales prostáticas, pero también en testículo, cerebro, hígado, corazón y bazo ${ }^{13}$. El antígeno de células madre prostáticas (PSCA Prostate stem cell antigen) es el antígeno asociado a la próstata descubierto más recientemente; es una proteína de membrana tipo I de 123 aminoácidos sobreexpresada en más del $85 \%$ de todos los cánceres prostáticos. Tiene una especificidad tisular mayor que la del PSMA ${ }^{14}$. Todavía está en controversia una posible correlación entre la extensión de la expresión de PSCA y la puntuación de Gleason y/o el estadio clínico ${ }^{17,18}$. Puesto que la pérdida de PSCA desde la superficie celular es muy pequeña, este es posiblemente el AAT más prometedor en cáncer de próstata. Tres grupos han comunicado respuestas específicas de células T citotóxicas contra péptidos restringidos por HLAA2 in vitro ${ }^{17,19,20}$.

Otros potenciales antígenos asociados con tumores incluyen proteínas o carbohidratos que son altamente expresados en el cáncer de prósta$\mathrm{ta}^{21-23}$, antígenos de cáncer testicular ${ }^{23}$, y otros antígenos no específicos de la próstata como transcriptasa inversa de telomerasa (TERT: Telomerase reverse transcriptase $)^{25-27}$ y Her-2/neu ${ }^{28}$.

\section{CONSIDERACIONES METODOLÓGICAS EN ENSAYOS CLÍNICOS DE INMUNOTERAPIA EN CÁNCER DE PRÓSTATA}

Elección de la formulación farmacéutica de la vacuna

En la pasada década se ha desarrollado una amplia colección de estrategias de terapia inmune para cáncer de próstata. Una de las modalida- 
des de tratamiento estudiadas más extensivamente ha sido la vacunación con células dendríticas cargadas de antígeno tumoral. Las células dendríticas son células derivadas de la médula ósea que pueden captar y procesar antígenos. En un ambiente inflamatorio, las células dendríticas se activan y maduran con cambios fenotípicos y funcionales característicos. Estos cambios suponen una disminución de la capacidad de captación y procesado de antígenos, en paralelo con la expresión de moléculas de superficie de adhesión y coestimulación críticas para la activación de las células T. Después de la migración a los órganos linfoides el antígeno es presentado a las células $\mathrm{T}$ de forma restringida por el complejo mayor de histocompatibilidad (CMH). Las células dendríticas son las únicas células presentadoras de antígenos del sistema inmune capaces no sólo de disparar las respuestas de memoria sino también de iniciar las células $\mathrm{T}$ vírgenes. Cargadas con AAT pueden inducir respuestas de células $\mathrm{T}$ específicas de tumor tanto in vitro como in vivo ${ }^{29}$. Puesto que en condiciones de cultivo adecuadas in vitro se pueden generar en pocos días células dendríticas a partir de monocitos de sangre periférica, su papel crucial en la respuesta inmune las convierte en una de las plataformas más prometedoras para inmunoterapia antitumoral. Sin embargo, no todas las células dendríticas son inmunogénicas. La exposición al factor estimulante de colonias de granulocitos y monocitos (GM_CSF) y a IL-4 lleva a la diferenciación a células dendríticas inmaduras solamente. La maduración de las células dendríticas puede inducirse mediante estimulación del receptor CD40 o por sustancias que imiten un entorno infeccioso o inflamatorio, la mayoría de los cuales se unen a receptores de tipo toll (TLR- Toll-like receptors) ${ }^{30-32}$. Una alternativa es administrar células dendríticas inmaduras junto con adyuvantes que actúen a través de los TLR para inducir la maduración in situ de las células dendríticas ${ }^{33}$.

Varios grupos pudieron demostrar que las células dendríticas inmaduras pueden inhibir la inmunidad mediada por células $\mathrm{T}$ o incluso inducir tolerancia contra un determinado antígeno in vitro e in vivo ${ }^{34}$. En un metaanálisis de diez ensayos clínicos en pacientes con melanoma la evolución fue mejor con células dendríticas maduras ${ }^{35}$.
Finalmente, en estudios comparativos entre células dendriticas inmaduras y maduras cargadas con péptidos, solamente las células dendríticas maduras fueron capaces de inducir inmunidad antígeno-especifica y respuestas clínicas en pacientes con melanoma, vacunados ${ }^{36,37}$. Por lo tanto, se ha sugerido que las células dendríticas óptimas apropiadas para vacunación deberían tener un fenotipo maduro, indicado por la expresión de CD83, retener sus habilidades migratorias, y preferiblemente segregar citoquinas promotoras de TH1, tales como IL-12p70, y citoquinas no inmunosupresoras como IL- $10^{38}$.

Puesto que no existe un estándar internacional de maduración de células dendríticas, son esenciales controles de calidad de las células dendríticas utilizadas para vacunación, y una completa caracterización fenotípica y funcional debería ser incluida en la comunicación del ensayo clínico para permitir la interpretación de los resultados a la luz del tipo de células dendríticas utilizada.

Para inducir una respuesta inmune específica contra tumor, las células dendriticas necesitan presentar AAT. Hay varias posibilidades de aportación de antígenos. El método más ampliamente utilizado hoy en día para vacunación con células dendríticas consiste en pulsar las células dendríticas con péptidos tumorales específicos que son presentados por el $\mathrm{CMH}$ apropiado. Una de las ventajas de la utilización de péptidos es un epítopo bien descrito que permite la producción estandarizada de la vacuna y una monitorización inmune exacta. Alternativamente, las células dendríticas pueden ser cargadas con proteínas completas o con una combinación de proteínas. La proteína completa es procesada de forma natural por la celular dendrítica y ésta presentará un amplio espectro de péptidos. La vida media del complejo péptido-CMH es mayor que después de una carga exógena de péptidos. Finalmente, las preparaciones con células tumorales enteras tienen la ventaja de que no es necesario conocer el antígeno inmunogénico, aunque la disponibilidad de material tumoral limita este abordaje. Algunos investigadores han utilizado líneas de células tumorales alogénicas lisadas. La utilización de células alogénicas implica problemas potenciales porque existe la posibilidad de que 
les falten algunos de los antígenos de tumor del paciente y que por otro lado contengan antígenos irrelevantes o incluso antígenos no deseados como aloantígenos y antígenos menores de histocompatibilidad.

En vez de cargar las células dendríticas in vitro antes de la inmunización, se pueden inyectar lisados de células tumorales, proteínas AAT o fragmentos peptídicos de AAT restringidas por $\mathrm{CMH}$ con adyuvantes químicos o biológicos adecuados. Entonces las células dendríticas residentes capturan, procesan (en el caso de proteínas completas) y presentan el antígeno in vivo. Alternativamente, se pueden aportar genes de AAT en una forma inmunogénica, como vacunas de DNA o vectores virales creados por ingeniería genética. Los vectores virales frecuentemente inducen potentes respuestas inmunes antivirales las cuales pueden evitar la administración repetida.

\section{Elección de los criterios clínicos de evaluación}

Un problema frecuente en los ensayos clínicos en cáncer de próstata es la falta de enfermedad mensurable. Sin embargo, la significación clínica de los cambios del PSA sérico ha sido demostrada en múltiples estudios, y un descenso del PSA $\geq 50 \%$ después del tratamiento es un factor independiente asociado con la supervivencia en pacientes con enfermedad metastásica ${ }^{39-42}$. Por lo tanto, se ha recomendado el PSA como un marcador indirecto de los criterios de evaluación de resultados clínicos y se ha incorporado dentro de los criterios de remisión en pacientes con enfermedad metastásica $^{43}$. Sin embargo, la situación de los pacientes con recurrencia bioquímica, definida como una recurrencia aislada del PSA sin ninguna otra evidencia de enfermedad activa, no está tan bien examinada. El grupo de trabajo de PSA recomendó que en los ensayos clínicos en pacientes con recidiva bioquímica fueran definidos los criterios de remisión basados en PSA, aunque los criterios de valoración clínica tales como supervivencia cáncer de próstata específica deberian utilizarse preferentemente cuando fuera posible. Aunque éstos son criterios de valoración relevantes en cáncer de próstata avanzado, alcanzarlos puede llevar un tiempo demasiado largo en ensayos clínicos de recidiva bioquímica, en los que se ha sugerido el tiempo hasta el desarrollo de metástasis como una alternativa $^{44}$. En pacientes con recidiva bioquímica, se ha descrito que sin tratamiento el PSA aumenta siguiendo un patrón logarítmico-lineal con el tiempo ${ }^{45}$, y que los cambios en la cinética del PSA, como la pendiente o el tiempo de duplicación de PSA (PSA-DT) tienen correlación con la muerte por cáncer de próstata después de prostatectomía ${ }^{46}$. Sin embargo, la modulación de la cinética del PSA con tratamiento sistémico no ha mostrado todavía correlación con un beneficio clínico para el paciente. No obstante, en una revisión reciente, se ha sugerido el cambio del PSADT como criterio de evaluación en ensayos clínicos de recidiva bioquímica ${ }^{47}$. En general, las diferencias en los criterios de valoración clínica e incluso de los criterios de remisión hacen que sean difíciles las comparaciones de los resultados de los estudios en cáncer de próstata.

\section{Monitorización inmunológica}

El objetivo primario de la inmunoterapia es la inducción de una respuesta inmune celular o humoral específica contra el tumor. Los anticuerpos antitumorales son relativamente fáciles de medir con ELISA. Las células $\mathrm{T}$ antígeno específicas pueden ser cuantificadas por citometría de flujo con multímeros HLA cargados con péptidos, marcados mediante fluorescencia. Los ensayos funcionales con linfocitos $\mathrm{T}$ in vitro incluyen proliferación (linfocitos $\mathrm{T} \mathrm{CD}^{+}$), ensayos de muerte de células diana mediante la liberación de $\mathrm{Cr}^{51}$ (linfocitos $\mathrm{T}$ citotóxicos), o la detección de secreción de citoquinas (IFN $\gamma$ ) después de la estimulación con antígenos, por ejemplo por tinción de citoquinas intracelulares o ELIspot. Se han utilizado las reacciones de hipersensibilidad de tipo diferido para demostrar respuestas inmunes in vivo, y es posible analizar la especificidad antigénica de las células $\mathrm{T}$ infiltrantes. Sin embargo, existen diferentes métodos para cada uno de estos ensayos y la correlación entre las diferentes pruebas es variable. Más aún, la monitorización inmune no ha sido frecuentemente realizada en los ensayos clínicos sin células dendríticas. 


\section{ENSAYOS CLÍNICOS DE INMUNOTERAPIA EN CÁNCER DE PRÓSTATA \\ Protocolos de inmunización basados en células dendriticas}

El primero de una serie de estudios de vacunación con células dendríticas en cáncer de próstata utilizó péptidos derivados de PSMA restringidos por HLA-A2 ${ }^{48}$. Sin embargo, excepto por $1 / 46$ pacientes en seguimiento que tuvo una reacción positiva de hipersensibilidad de tipo diferido, no se pudieron inducir linfocitos $\mathrm{T}$ citotóxicos específicos contra péptidos de PSMA, e incluso tres de los respondedores eran HLA-A2 negativos ${ }^{48-50}$. Otro grupo ha comunicado recientemente linfocitos $\mathrm{T}$ $\mathrm{CD}^{+}$específicos contra PSMA en 3/4 respondedores en un ensayo clínico se aplicaba cinco péptidos derivados de diferentes $\mathrm{AAT}^{51}$.

Aunque el PSA es una proteína circulante, lo cual hace de la tolerancia periférica PSA-especifica un probable mecanismo de escape tumoral, en cada uno de los seis ensayos clínicos de vacunación con células dendríticas utilizando PSA se indujeron respuestas inmunes específicas de péptido o proteína (en conjunto 49 de 99 pacientes, rango $17-100 \%)^{51-57}$.

Cargando una proteína de fusión FAP-GM-CSF (PROVENGE) en una población de células mononucleares de sangre periférica enriquecida en células presentadoras de antígeno se demostraron respuestas inmunes contra FAP en el 38\% de los pacientes $^{58}$. Utilizando el mismo abordaje aunque con un esquema distinto de aplicación se comunicaron respuestas inmunes celulares contra FAP y GM-CSF en 9/9 pacientes evaluables ${ }^{59}$. El primer ensayo clínico randomizado con 127 pacientes demostró una ventaja de supervivencia en los pacientes tratados con inmunoterapia frente a placebo $^{60}$. En otro abordaje interesante, las células dendríticas fueron cargadas con FAP murina, como un xenoantígeno presumiblemente más inmunogénico que él propio ${ }^{61}$. Se pudo demostrar respuesta de linfocitos T contra FAP murina en cada uno de los 21 pacientes, en 11 de ellos contra la FAP humana.

La inmunización con células dendríticas y péptidos restringidos por HLA-A2 obtuvo respuestas inmunes específicas contra PSCA en la mitad de los pacientes tratados ${ }^{55,56}$.
En un estudio con 11 pacientes con recidiva bioquímica inmunizados con células dendríticas cargadas con un lisado de células tumorales alogénicas, se comunicaron respuestas inmunes celulares en 10 y estabilización del PSA en $8^{62}$. Posiblemente el abordaje más elegante es la trasfección de células dendríticas con mRNA codificante para $\mathrm{AAT}^{27,52}$ o con RNA extraído de líneas celulares de tumor ${ }^{63}$. Como los AAT son procesados endógenamente, las respuestas inmunes resultantes fueron predominantemente mediadas por linfocitos $\mathrm{T} \mathrm{CD}^{+}$, aunque también se observaron algunas respuestas de linfocitos $\mathrm{T} \mathrm{CD} 4^{+}$.

En conjunto, la vacunación con células dendríticas pudo inducir respuestas inmunes celulares específicas de antígeno en el $67 \%$ de los pacientes (142/213) comunicados, incluyendo hipersensibilidad de tipo diferido, ELISpot, análisis de tetrámeros o proliferación de linfocitos $\mathrm{T}$. En varios grupos se observó una correlación entre las respuestas inmune y clínica: en un ensayo clínico con células dendríticas cargadas con péptidos, se detectaron respuestas de linfocitos $T$ específicas de péptido en $4 / 8$ pacientes, de los cuales 3 también tuvieron una respuesta clínica $^{51}$. La vacunación con células dendríticas transfectadas con RNAm de lineas celulares indujo respuestas inmunes en $10 / 11$ pacientes con enfermedad estable, pero sólo en $3 / 8$ con enfermedad progresiva ${ }^{63}$. Se ha observado correlación entre respuestas inmunes celulares y tiempo a progresión ${ }^{60}$ y supervivencia ${ }^{55}$. El único ensayo clínico randomizado controlado con placebo de vacunación con células dendríticas en cáncer de próstata demostró un beneficio de supervivencia de 4,5 meses $(25,9$ vs. 21,4$)$ en la cohorte de vacunación ${ }^{60}$. Otros grupos comunicaron enfermedad estable por hallazgos radiológicos ${ }^{26,61}$, pero en la mayoría de los estudios los criterios de remisión y respuesta al tratamiento se basaron en valores de PSA o cambios en la cinética del PSA. Se consiguió estabilización de la enfermedad (incluyendo cambios en la pendiente del PSA) entre el 10\% (3/31) y el 100\% (4/4), o en conjunto el 45\% (78/175) de los pacientes tratados con células dendríticas. Se comunicaron remisiones parciales en el 16\% (24/147, rango 10$27 \%$ ). Solamente se observó una remisión completa en un estudio de seguimiento con células den- 
dríticas cargadas con péptidos de PSMA en pacientes con cáncer de próstata andrógeno independiente $(\mathrm{CPAI})^{64}$.

\section{Vacunas de células completas y basadas en adyuvantes}

En un abordaje individualizado, se inmunizaron pacientes con enfermedad metastásica utilizando péptidos identificados de linfocitos $\mathrm{T}$ citotóxicos circulantes preexistentes junto con el adyuvante de Freund incompleto (IFA- Incomplete Freund`s adjuvant). El número de linfocitos $\mathrm{T}$ citotóxicos aumentó después del tratamiento en $4 / 10$ pacientes HLA-A2 ${ }^{+}$y HLA-A2 $4^{+}$respectivamente, mientras que se pudieron observar efectos clínicos en 6/10 pacientes HLA-A $24^{+}$ pero sólo en 3/10 HLA-A2 ${ }^{+65,66}$. El adyuvante de saponina QS21 y la proteína activadora de linfocitos T Helper Hemocianina de lapa han sido utilizadas juntas con éxito en varios ensayos clinicos en pacientes con recidiva bioquímica. Se utilizaron como antígenos diferentes carbohidratos altamente expresados en cáncer, como Globo $\mathrm{H}$, MUC-2, el monosacárido sintético Tn (c) y el antígeno de Thomson-Friedrich ${ }^{23,67-69}$. Se observaron respuestas con IgG e IgM contra los antígenos de la vacuna en la mayoría de los pacientes, y también se comunicó estabilización o reducción de la pendiente del PSA, en algunos casos duradera (2 años).

El MUC-1 es otro carbohidrato diana en inmunoterapia en pacientes con recidiva bioquímica. Una vacuna liposomal con el MUC-1 y un adyuvante de base lipídica, precedida por una inyección única de $300 \mathrm{mg} / \mathrm{m}^{2}$ de ciclofosfamida indujo una estabilización o disminución del PSA en $8 / 16$ pacientes $^{70}$.

Además del IFA y otras formulaciones de base lipídica, se utiliza frecuentemente como adyuvante el factor estimulante de colonias de granulocitos-macrófagos (GM_CSF). También se han visto efectos biológicos transitorios cuando se aplica solo, pero, excepto por un paciente con una mejoría en la gammagrafía ósea, se limitaron a modulaciones transitorias del $\mathrm{PSA}^{71-74}$. Her-2/neu es un protooncogén expresado en muchos cánceres de células epiteliales. Se administraron un péptido de Her-2/neu restringido por HLA-A2 y GMCSF como tratamiento adyuvante en pacientes de alto riesgo, utilizando pacientes HLA-A2 negativos como controles ${ }^{28}$. Se pudieron inducir linfocitos $\mathrm{T} \mathrm{CD}^{+}$específicos de péptido en los 7 pacientes vacunados, y a pesar de que los pacientes HLA-A2 ${ }^{+}$tenían una puntuación de riesgo mayor la supervivencia libre enfermedad fue comparable en ambos grupos. En un ensayo clínico con nueve pacientes con CPAI se utilizaron plásmidos que expresaban PSA junto con GM-CSF e IL2 ${ }^{75}$. Solamente 2/8 tuvieron una disminución de la pendiente del PSA y sólo se detectaron linfocitos $\mathrm{T}$ citotóxicos en $2 / 3$ pacientes tratados en el nivel de dosis más alto, pero es interesante que las respuestas inmunes celulares se correlacionaron con estabilización clínica. Los efectos ayudantes del GM-CSF se han utilizado también en una vacuna con células tumorales enteras. En estudios previos las células tumorales del paciente fueron transducidas con DNAc codificando GM-CSF ${ }^{76}$. Para permitir la preparación de vacunas a gran escala, hoy se están utilizando en su lugar líneas de células tumorales alogénicas transducidas con GM-CSF. En un ensayo clínico con 21 pacientes con recidiva bioquímica se consiguió una reducción de la pendiente del PSA en el $76 \%$ de los pacientes y se detectaron anticuerpos contra los antígenos prostáticos ${ }^{77}$. Una combinación de tres líneas celulares de cáncer de próstata sin modificar condujo a un descenso de las velocidades de PSA en 11/26 pacientes con CPAI y a un tiempo largo hasta la progresión de 58 semanas $^{78}$.

\section{VACUNAS RECOMBINANTES BASADAS EN VIRUS}

La vacuna recombinante (rV) y la vacuna basada en el virus de la viruela aviar (Recombinant Fowlpox- rF) se han desarrollado en los últimos ocho años. La vacuna inicial contenía una rV que expresaba PSA, administrada con o $\sin \mathrm{GM}-\mathrm{CSF}^{79,80}$. Ensayos clínicos posteriores evaluaron el mejor régimen de tratamiento, consistente en una vacunación inicial de base con rV-PSA, seguida por varios refuerzos con $\mathrm{rF}$, para prevenir el desarrollo de anticuerpos antivacuna que interferirían con sus propiedades inmunológicas $^{81,82}$.

En estudios más tardíos se añadió en la fase de iniciación un virus de la vacuna que expresa- 
ba la molécula B7.1 (CD80), coestimulante de linfocitos T. Esta vacuna, complementada con GMCSF e IL-2, fue probada en un ensayo clínico randomizado en pacientes con enfermedad localizada tratados con radioterapia frente a radioterapia aislada $^{81,82}$. En 13/17 pacientes, los linfocitos T PSAespecíficos se expandieron después de la vacuna, y también se desarrollaron respuestas de linfocitos $\mathrm{T}$ contra otros AAT prostáticos, sin observarse efectos inmunológicos en el brazo de radioterapia aislada. Un ensayo clínico similar comparó nilutamida, un antiandrógeno, con o sin la vacuna referida en pacientes con CPAI no metastásico. Se indujeron linfocitos T PSA-específicos en 4/8 pacientes en el brazo de la vacuna en comparación con ningún caso del brazo de tratamiento convencional. El tiempo al fracaso del tratamiento era comparable en ambos brazos, pero se prolongó en aquellos pacientes que cambiaron a nilutamida después de la vacunación en comparación con nilutamida sola. Se evaluó la misma vacuna con docetaxel frente a vacuna aislada en un estudio randomizado en pacientes con CPAI metastásico ${ }^{84}$. La mediana del aumento de linfocitos T PSA-específicos productores de IFN $\gamma$ fue idéntica en ambos brazos, mientras que la mediana de supervivencia libre de progresión con docetaxel después de vacuna fue superior a la de controles históricos. Generaciones posteriores de la vacuna incluyeron la adición de secuencias génicas codificando las moléculas de adhesión ICAM-1 (CD54) y LFA-3 (CD58;TRICOM $\left.{ }^{\circledR}\right)$. El tratamiento de 10 pacientes con rV-PSA y refuerzos con $\mathrm{rF}$-PSA y TRICOM ${ }^{\circledR}$ condujo a estabilización de la enfermedad (PSA) en 4 e indujo anticuerpos contra la vacuna en todos $^{85}$.

\section{DESAFÍOS Y DIRECCIONES FUTURAS}

La experiencia disponible demuestra que la tasa global de respuestas de la inmunoterapia activa en cáncer de próstata no es inferior que la de quimioterapia y se asocia con una tolerancia bastante mejor. Sin embargo, la mayoría de respuestas clínicas parecen ser modestas: sólo se ha comunicado un caso de remisión completa, y la duración de la respuesta, cuando ha sido comunicada, estaba en el rango de semanas más que en el de meses en la mayoría de los casos. Varios factores pueden contribuir a aumentar la eficacia de la terapia de vacunación:
1. Competencia inmunológica del paciente: todos los estudios excepto uno se llevaron a cabo en pacientes con enfermedad avanzada. Estos pacientes estaban inmunocomprometidos por tratamientos previos y por el tumor mismo. Por lo tanto, la vacunación de pacientes inmunocompetentes con baja carga tumoral, en un escenario de adyuvancia o en estado de recidiva bioquímica podría ser una estrategia más prometedora. En pacientes con recidiva bioquímica, se comunicaron disminuciones del PSA en $11 / 24$ pacientes y respuestas de linfocitos $\mathrm{T}$ de nuevo desarrollo en $4 / 24$, a pesar de utilizar células dendríticas inmaduras ${ }^{53}$. Los 6 pacientes con células tumorales circulantes tuvieron una prueba de PCR negativa después de la vacunación.

2. Formulación de la vacuna: Pequeños ensayos clínicos en fase I/II han evaluado diferentes métodos de preparación de las células dendríticas, diferentes vías de administración y diferentes fuentes de antígenos. 11/17 ensayos clínicos aquí referidos utilizaron células dendríticas inmaduras. Puesto que las células dendríticas inmaduras pueden inducir tolerancia, los resultados pueden posiblemente mejorarse con la maduración de las células dendríticas. Son obligatorios los controles de calidad estrictos y la comunicación del fenotipo, el perfil de citoquinas y la función in vitro de las células dendríticas utilizadas en los ensayos clínicos.

3. Elección de antígeno: Posiblemente la elección del antígeno explica los modestos resultados clínicos en comparación con los ensayos clínicos en pacientes con melanoma. La inmunogenicidad de los AAT prostáticos es presumiblemente bastante baja. Adicionalmente, el número de linfocitos $\mathrm{T}$ vírgenes precursores próstataespecíficos en sangre periférica puede ser varios órdenes de magnitud menor que los melanomaespecíficos. La utilización de los llamados péptidos heteroclíticos con una fuerza de unión al $\mathrm{CMH}$ aumentada podría mejorar la inmunogenicidad de los péptidos ${ }^{86}$.

4. Combinación con modalidades de tratamiento convencional: La combinación de dosis tumoricidas de quimioterapia o radiación y vacunas puede ser ventajosa porque las células tumorales 
que mueren suministran antígenos tumorales en un ambiente inflamatorio, que sucesivamente pueden disparar la maduración de células dendríticas in vivo, contribuyendo a las respuestas inmunes inducidas por la vacuna.

5. Eliminación de los linfocitos T reguladores: los linfocitos $\mathrm{T}$ reguladores (Treg) se caracterizan por la expresión simultánea de CD4 y CD25 en su superficie y por la transcripción del factor Foxp3.

Los Treg suprimen la función de los linfocitos $\mathrm{T} \mathrm{CD}^{+}$de una manera dependiente del contacto celular. Su función fisiológica parece ser el control de la autoinmunidad en la periferia ${ }^{87}$. Adicionalmente, los Treg se expanden frecuentemente en pacientes portadores de tumores y pueden así inhibir eficientemente las respuestas inmunes celulares antitumorales ${ }^{88}$.

Consecuentemente, la inducción de Treg durante la vacunación o su expansión ya presente en el paciente son un posible factor contribuyente para una menor eficacia de la vacuna. Por lo tanto, la combinación de la eliminación de Treg e inmunoterapia parece un abordaje terapéutico prometedor.

Cuando se compara la vacunación con células dendríticas en pacientes con carcinoma de células renales con o sin eliminación previa de Treg por medio de un conjugado de toxina de difteria e IL2 (ONTAK), se consigue una tasa de respuesta inmune superior en el grupo ONTAK ${ }^{89}$.

Dosis bajas (es decir, no tumoricidas) de quimioterapia puede también actuar sinérgicamente con la vacuna: se podría demostrar que dosis bajas de ciclofosfamida disminuyen selectivamente el número y la función de los Treg con poco efecto sobre otras subpoblaciones de linfocitos $^{90-92}$. En pacientes con melanoma metastásico refractario, la combinación de ciclofosfamida y fludarabina administrada antes de la infusión de linfocitos de tumor infiltrante dio como resultado respuestas clínicas dramáticas, incluyendo tres remisiones completas ${ }^{93}$. Sin embargo, los pacientes recibieron quimioterapia a dosis normales con el resultado de toxicidad esperado, así como dos casos de neumonía por Pneumocystis y uno de enfermedad linfoproliferativa relacionada con el virus de Epstein-Barr. En otro ensayo clínico, dosis bajas de ciclofosfamida que inducían toxici- dad insignificante sólo fueron capaces de aumentar la inmunogenicidad de la vacuna en pacientes con carcinoma de células renales ${ }^{94}$.

\section{CONCLUSIONES}

De los más de 50 ensayos clínicos de inmunoterapia publicados en revistas científicas con comités de revisión todos sugieren que la inmunoterapia activa en cáncer de próstata es segura. Los efectos colaterales se han restringido principalmente a reacciones locales en el sitio de inyección y reacciones generales leves como fiebre, especialmente en ensayos clínicos que utilizaban GM-CSF o IL-2. Adicionalmente, el riesgo de inducir autoinmunidad, que pueda aparecer, como por ejemplo el vitíligo después de la vacuna en melanoma, y pueda estar asociada con respuestas clínicas ${ }^{95}$ puede ser aceptable dado el hecho de que la próstata representa esencialmente un tejido prescindible.

La frecuencia de respuestas inmunes y cambios favorables en los parámetros clínicos tales como reducciones de la cinética de PSA variaron en los ensayos clínicos. Con vacunas basadas en células dendríticas, se vieron efectos biológicos en cerca del $45 \%$ de los pacientes. Éstas fueron subrayadas principalmente por respuestas inmunes mediadas por linfocitos $\mathrm{T}$ y en algunos casos humorales en cerca de $67 \%$ de los pacientes. La comparación de diferentes estrategias de vacunación es casi imposible debido a las grandes diferencias en los criterios de selección de los pacientes y los criterios de evaluación. En muchos ensayos clínicos, especialmente aquellos sin células dendríticas, la presentación de la monitorización inmune es insuficiente, haciendo difícil la interpretación de los datos, e incluso las comparaciones informales entre ensayos clínicos son imposibles. La evolución de los pacientes parece ser similar con diferentes estrategias de vacunación, pero las frecuencias de pacientes que responden y de respuestas biológicas duraderas parecen ser de alguna forma superiores con formulaciones basadas en virus o células dendríticas en comparación con aquellas basadas en células tumorales adyuvantes, naturales o manipuladas por ingeniería genética.

En varios estudios, se observó correlación entre las respuestas inmunes y los efectos clíni- 
cos. En el único ensayo clínico de vacunación fase III randomizado y controlado con placebo se pudo demostrar un beneficio de supervivencia de varios meses en los pacientes vacunados ${ }^{60}$. Aunque los otros ensayos clínicos (fases I/II) no pudieron suministrar pruebas formales de eficacia clinica, sus resultados son estimulantes y suministran una prueba de principio de la inmunogenicidad de la vacunación antitumoral en pacientes con cáncer de próstata. Para mejorar más aún los resultados con los pacientes se están explorando varias estrategias que afectan a la elección del antígeno o la optimización de la formulación de la vacuna (por ejemplo mejorando la maduración de las células dendríticas o desarrollando vectores altamente inmunogénicos).

La combinación con tratamiento convencional o la modulación inmunológica sistémica (como la eliminación de Treg) son abordajes adicionales que buscan mejorar la eficacia de la inmunización. Finalmente los pacientes con baja carga tumoral y mejor competencia inmunológica podrían beneficiarse más de la terapia de vacunación que los pacientes con cáncer en estadios avanzados con fuertes tratamientos previos.

\section{Agradecimientos}

Este trabajo ha recibido una beca de investigación de la Fundación Wilhelm-Sander, Alemania (a A.-K. T.$\mathrm{K}$ y H.V). Los autores declaran que no tienen conflictos de intereses potenciales.

\section{REFERENCIAS}

1. Jemal A, Murray T, Ward E, Samuels A, Tiwari RC, Ghafoor A, Feuer EJ, Thun MJ. Cancer statistics, 2005. CA Cancer J Clin 2005;55(1): 10-30.

2. Ferlay J, Autier P, Boniol M, Heanue M, Colombet M, Boyle P. Estimates of the cancer incidence and mortality in Europe in 2006. Ann Oncol 2007;18(3):581-592.

3. Wetterauer U, Rutishauser G, Sommerkamp H. Urologie: de Gruyter, 1995:225-233[

4. Pound CR, Partin AW, Eisenberger MA, Chan DW, Pearson JD, Walsh PC. Natural history of progression after PSA elevation following radical prostatectomy. Jama 1999;281(17):1591-1597.

5. Maximum androgen blockade in advanced prostate cancer: an overview of 22 randomised trials with 3283 deaths in 5710 patients. Prostate Cancer Trialists' Collaborative Group. Lancet. 1995 Jul 29;346(8970):265-269.

6. Petrylak DP, Tangen CM, Hussain MH, Lara PN, Jr., Jones JA, Taplin ME, Burch PA, Berry D, Moinpour C, Kohli M, Benson MC, Small EJ, et al. Docetaxel and estramustine compared with mitoxantrone and prednisone for advanced refractory prostate cancer. N Engl J Med 2004;351(15):1513-1520.
7. Tannock IF, de Wit R, Berry WR, Horti J, Pluzanska A, Chi KN, Oudard S, Theodore C, James ND, Turesson I, Rosenthal MA, Eisenberger MA. Docetaxel plus prednisone or mitoxantrone plus prednisone for advanced prostate cancer. N Engl J Med 2004;351(15):1502-1512.

8. Oesterling JE. Prostate specific antigen: a critical assessment of the most useful tumor marker for adenocarcinoma of the prostate. J Urol 1991;145:907-23.

9. Xue BH, Zhang Y, Sosman JA, Peace DJ. Induction of human cytotoxic T lymphocytes specific for prostate-specific antigen. Prostate 1997;30(2):73-78.

10. Correale P, Walmsley K, Nieroda C, Zaremba S, Zhu M, Schlom J, Tsang KY. In vitro generation of human cytotoxic $\mathrm{T}$ lymphocytes specific for peptides derived from prostatespecific antigen. J Natl Cancer Inst 1997;89(4):293-300.

11. Correale P, Walmsley K, Zaremba S, Zhu M, Schlom J, Tsang KY. Generation of human cytolytic T lymphocyte lines directed against prostate-specific antigen (PSA) employing a PSA oligoepitope peptide. J Immunol 1998; 161(6):3186-3194.

12. Renneberg H, Friedetzky A, Konrad L, Kurek R, Weingartner K, Wennemuth G, Tunn UW, Aumuller G. Prostate specific membrane antigen (PSM) is expressed in various human tissues: implication for the use of PSM reverse transcription polymerase chain reaction to detect hematogenous prostate cancer spread. Urol Res 1999; 27(1):23-27.

13. Heller JE. Prostatic acid phosphatase: its current clinical status. J Urol 1987;137(6):1091-103.

14. Reiter RE, Gu Z, Watabe T, Thomas G, Szigeti K, Davis E, Wahl M, Nisitani S, Yamashiro J, Le Beau MM, Loda M, Witte ON. Prostate stem cell antigen: a cell surface marker overexpressed in prostate cancer. Proc Natl Acad Sci U S A 1998;95(4): 1735-1740.

15. Gu Z, Thomas G, Yamashiro J, Shintaku IP, Dorey F, Raitano A, Witte ON, Said JW, Loda M, Reiter RE. Prostate stem cell antigen (PSCA) expression increases with high gleason score, advanced stage and bone metastasis in prostate cancer. Oncogene 2000;19(10):1288-1296.

16. Han KR, Seligson DB, Liu X, Horvath S, Shintaku PI, Thomas GV, Said JW, Reiter RE. Prostate stem cell antigen expression is associated with gleason score, seminal vesicle invasion and capsular invasion in prostate cancer. $\mathrm{J}$ Urol 2004;171(3):1117-1121.

17. Dannull J, Diener PA, Prikler L, Furstenberger G, Cerny T, Schmid U, Ackermann DK, Groettrup M. Prostate stem cell antigen is a promising candidate for immunotherapy of advanced prostate cancer. Cancer Res 2000;60(19):55225528.

18. Ross S, Spencer SD, Holcomb I, Tan C, Hongo J, Devaux B, Rangell L, Keller GA, Schow P, Steeves RM, Lutz RJ, Frantz G, et al. Prostate stem cell antigen as therapy target: tissue expression and in vivo efficacy of an immunoconjugate. Cancer Res 2002;62(9):2546-2553.

19. Kiessling A, Schmitz M, Stevanovic S, Weigle B, Holig K, Fussel M, Fussel S, Meye A, Wirth MP, Rieber EP. Prostate stem cell antigen: Identification of immunogenic peptides and assessment of reactive CD8+ T cells in prostate cancer patients. Int J Cancer 2002(4); 102:390-397.

20. Matsueda S, Kobayashi K, Nonaka Y, Noguchi M, Itoh K, Harada M. Identification of new prostate stem cell antigenderived peptides immunogenic in HLA-A2(+) patients with hormone-refractory prostate cancer. Cancer Immunol Immunother 2004;53(6):479-89. 
21. Myers RB, Meredith RF, Schlom J, LoBuglio AF, Bueschen AJ, Wheeler RH, Stockard CR, Grizzle WE. Tumor associated glycoprotein-72 is highly expressed in prostatic adenocarcinomas. J Urol 1994;152(1):243-246.

22. Slovin SF, Scher HI. Peptide and carbohydrate vaccines in relapsed prostate cancer: immunogenicity of synthetic vaccines in man-clinical trials at Memorial Sloan-Kettering Cancer Center. Semin Oncol 1999;26(4):448-454.

23. Slovin SF, Ragupathi G, Fernandez C, Jefferson MP, Diani M, Wilton AS, Powell S, Spassova M, Reis C, Clausen H, Danishefsky S, Livingston P, et al. A bivalent conjugate vaccine in the treatment of biochemically relapsed prostate cancer: a study of glycosylated MUC-2-KLH and Globo $\mathrm{H}-\mathrm{KLH}$ conjugate vaccines given with the new semi-synthetic saponin immunological adjuvant GPI-0100 OR QS21. Vaccine 2005;23(4):3114-3122.

24. Jager D, Karbach J, Pauligk C, Seil I, Frei C, Chen YT, Old W, Knuth A, Jager E. Humoral and cellular immune responses against the breast cancer antigen NY-BR-1: definition of two HLA-A2 restricted peptide epitopes. Cancer Immun 2005;5:11.

25. Nair SK, Heiser A, Boczkowski D, Majumdar A, Naoe M, Lebkowski JS, Vieweg J, Gilboa E. Induction of cytotoxic T cell responses and tumor immunity against unrelated tumors using telomerase reverse transcriptase RNA transfected dendritic cells. Nat Med 2000;6:1011-1017.

26. Vonderheide RH, Domchek SM, Schultze JL, George DJ, Hoar KM, Chen DY, Stephans KF, Masutomi K, Loda M, Xia Z, Anderson KS, Hahn WC, et al. Vaccination of cancer patients against telomerase induces functional antitumor CD8+ T lymphocytes. Clin Cancer Res 2004;10(3):828839.

27. Su Z, Dannull J, Yang BK, Dahm P, Coleman D, Yancey D, Sichi S, Niedzwiecki D, Boczkowski D, Gilboa E, Vieweg J. Telomerase mRNA-transfected dendritic cells stimulate antigen-specific $\mathrm{CD} 8+$ and $\mathrm{CD} 4+\mathrm{T}$ cell responses in patients with metastatic prostate cancer. J Immunol 2005; 174(6):3798-3807.

28. Hueman MT, Dehqanzada ZA, Novak TE, Gurney JM, Woll MM, Ryan GB, Storrer CE, Fisher C, McLeod DG, Ioannides CG, Ponniah S, Peoples GE. Phase I clinical trial of a HER-2/neu peptide (E75) vaccine for the prevention of prostate-specific antigen recurrence in high-risk prostate cancer patients. Clin Cancer Res 2005;11(2):7470-9.

29. Banchereau J, Steinman RM. Dendritic cells and the control of immunity. Nature 1998;392(6673):245-252.

30. Kawai T, Akira S. Pathogen recognition with Toll-like receptors. Curr Opin Immunol 2005;17(4):338-44.

31. Kalinski P, Vieira PL, Schuitemaker JH, de Jong EC, Kapsenberg ML. Prostaglandin E(2) is a selective inducer of interleukin-12 p40 (IL-12p40) production and an inhibitor of bioactive IL-12p70 heterodimer. Blood 2001;97(11): 3466-3469.

32. Harizi H, Juzan M, Pitard V, Moreau JF, Gualde N. Cyclooxygenase-2-issued prostaglandin e(2) enhances the production of endogenous IL-10, which down-regulates dendritic cell functions. J Immunol 2002;168(5):22552263.

33. Nair S, McLaughlin C, Weizer A, Su Z, Boczkowski D, Dannull J, Vieweg J, Gilboa E. Injection of immature dendritic cells into adjuvant-treated skin obviates the need for ex vivo maturation. J Immunol 2003;171(11):6275-6282.

34. Steinman RM, Hawiger D, Nussenzweig MC. Tolerogenic dendritic cells. Annu Rev Immunol 2003;21:685-711.
35. McIlroy D, Gregoire M. Optimizing dendritic cell-based anticancer immunotherapy: maturation state does have clinical impact. Cancer Immunol Immunother 2003;52(10): 583-91.

36. Jonuleit H, Giesecke-Tuettenberg A, Tuting T, ThurnerSchuler B, Stuge TB, Paragnik L, Kandemir A, Lee PP, Schuler G, Knop J, Enk AH. A comparison of two types of dendritic cell as adjuvants for the induction of melanomaspecific $\mathrm{T}$-cell responses in humans following intranodal injection. Int $J$ Cancer 2001;93(2):243-251.

37. de Vries IJ, Lesterhuis WJ, Scharenborg NM, Engelen LP, Ruiter DJ, Gerritsen MJ, Croockewit S, Britten CM, Torensma R, Adema GJ, Figdor CG, Punt CJ. Maturation of dendritic cells is a prerequisite for inducing immune responses in advanced melanoma patients. Clin Cancer Res 2003;9(14):5091-5100.

38. Figdor CG, de Vries IJ, Lesterhuis WJ, Melief CJ. Dendritic cell immunotherapy: mapping the way. Nat Med 2004;10 (5):475-480.

39. Small EJ, McMillan A, Meyer M, Chen L, Slichenmyer WJ, Lenehan PF, Eisenberger M. Serum prostate-specific antigen decline as a marker of clinical outcome in hormonerefractory prostate cancer patients: association with progression-free survival, pain end points, and survival. J Clin Oncol 2001;19(5):1304-1311.

40. Scher HI, Kelly WM, Zhang ZF, Ouyang P, Sun M, Schwartz M, Ding C, Wang W, Horak ID, Kremer AB. Posttherapy serum prostate-specific antigen level and survival in patients with androgen-independent prostate cancer. $\mathrm{J}$ Natl Cancer Inst 1999;91(3):244-251.

41. Smith DC, Dunn RL, Strawderman MS, Pienta KJ. Change in serum prostate-specific antigen as a marker of response to cytotoxic therapy for hormone-refractory prostate cancer. J Clin Oncol 1998;16(5):1835-1843.

42. Kelly WK, Scher HI, Mazumdar M, Vlamis V, Schwartz M, Fossa SD. Prostate-specific antigen as a measure of disease outcome in metastatic hormone-refractory prostate cancer. J Clin Oncol 1993;11:607-615.

43. Bubley GJ, Carducci M, Dahut W, Dawson N, Daliani D, Eisenberger M, Figg WD, Freidlin B, Halabi S, Hudes G, Hussain M, Kaplan R, et al. Eligibility and response guidelines for phase II clinical trials in androgen-independent prostate cancer: recommendations from the ProstateSpecific Antigen Working Group. J Clin Oncol 1999;17/ 11):3461-3467.

44. Scher HI, Eisenberger M, D’Amico AV, Halabi S, Small EJ, Morris M, Kattan MW, Roach M, Kantoff P, Pienta KJ, Carducci MA, Agus D, et al. Eligibility and outcomes reporting guidelines for clinical trials for patients in the state of a rising prostate-specific antigen: recommendations from the Prostate-Specific Antigen Working Group. J Clin Oncol 2004;22(3):537-556.

45. Schmid HP, McNeal JE, Stamey TA. Observations on the doubling time of prostate cancer. The use of serial prostate-specific antigen in patients with untreated disease as a measure of increasing cancer volume. Cancer 1993;71(6): 2031-2040.

46. D'Amico AV, Chen MH, Roehl KA, Catalona WJ. Preoperative PSA velocity and the risk of death from prostate cancer after radical prostatectomy. N Engl J Med 2004(5); 351:125-35.

47. Lin AM, Rini BI, Small EJ. Clinical trials in patients with biochemically relapsed prostate cancer. BJU Int 2006;97 (5):905-910. 
48. Murphy G, Tjoa B, Ragde H, Kenny G, Boynton A. Phase I clinical trial: $\mathrm{T}$-cell therapy for prostate cancer using autologous dendritic cells pulsed with HLA-A0201-specific peptides from prostate-specific membrane antigen. Prostate 1996(6);29:371-380.

49. Lodge PA, Jones LA, Bader RA, Murphy GP, Salgaller ML. Dendritic cell-based immunotherapy of prostate cancer: immune monitoring of a phase II clinical trial. Cancer Res 2000;60:829-833.

50. Tjoa BA, Simmons SJ, Bowes VA, Ragde H, Rogers M, Elgamal A, Kenny GM, Cobb OE, Ireton RC, Troychak MJ, Salgaller ML, Boynton AL, et al. Evaluation of phase I/II clinical trials in prostate cancer with dendritic cells and PSMA peptides. Prostate 1998;36(1):39-44.

51. Fuessel S, Meye A, Schmitz M, Zastrow S, Linne C, Richter K, Lobel B, Hakenberg OW, Hoelig K, Rieber EP, Wirth MP. Vaccination of hormone-refractory prostate cancer patients with peptide cocktail-loaded dendritic cells: results of a phase I clinical trial. Prostate 2006;66(8):811821 .

52. Heiser A, Coleman D, Dannull J, Yancey D, Maurice MA, Lallas CD, Dahm P, Niedzwiecki D, Gilboa E, Vieweg J. Autologous dendritic cells transfected with prostate-specific antigen RNA stimulate CTL responses against metastatic prostate tumors. J Clin Invest 2002;109:409-417.

53. Barrou B, Benoit G, Ouldkaci M, Cussenot O, Salcedo M, Agrawal S, Massicard S, Bercovici N, Ericson ML, Thiounn N. Vaccination of prostatectomized prostate cancer patients in biochemical relapse, with autologous dendritic cells pulsed with recombinant human PSA. Cancer Immunol Immunother 2004;53/5):453-460.

54. Perambakam S, Hallmeyer S, Reddy S, Mahmud N, Bressler L, Dechristopher P, Mahmud D, Nunez R, Sosman JA, Peace DJ. Induction of specific $\mathrm{T}$ cell immunity in patients with prostate cancer by vaccination with PSA146154 peptide. Cancer Immunol Immunother 2005(9):1-10.

55. Thomas-Kaskel AK, Zeiser R, Jochim R, Robbel C, Schultze-Seemann W, Waller CF, Veelken H. Vaccination of advanced prostate cancer patients with PSCA and PSA peptide-loaded dendritic cells induces DTH responses that correlate with superior overall survival. Int $J$ Cancer 2006;119(106):2428-2434.

56. Waeckerle-Men Y, Uetz-von Allmen E, Fopp M, von Moos R, Bohme C, Schmid HP, Ackermann D, Cerny T, Ludewig B, Groettrup M, Gillessen S. Dendritic cell-based multi-epitope immunotherapy of hormone-refractory prostate carcinoma. Cancer Immunol Immunother 2006; 55(12):152433.

57. Hildenbrand B, Sauer B, Kalis O, Stoll C, Freudenberg MA, Niedermann G, Giesler JM, Juttner E, Peters JH, Haring $\mathrm{B}$, Leo R, Unger C, et al. Immunotherapy of patients with hormone-refractory prostate carcinoma pre-treated with interferon-gamma and vaccinated with autologous PSApeptide loaded dendritic cells-A pilot study. Prostate 2007;67:(5)500-8.

58. Small EJ, Fratesi P, Reese DM, Strang G, Laus R, Peshwa MV, Valone FH. Immunotherapy of hormone-refractory prostate cancer with antigen-loaded dendritic cells. J Clin Oncol 2000;18(22):3894-903.

59. Burch PA, Breen JK, Buckner JC, Gastineau DA, Kaur JA, Laus RL, Padley DJ, Peshwa MV, Pitot HC, Richardson RL, Smits BJ, Sopapan P, et al. Priming tissue-specific cellular immunity in a phase I trial of autologous dendritic cells for prostate cancer. Clin Cancer Res 2000(6):2175-2182.
60. Small EJ, Schellhammer PF, Higano CS, Redfern CH, Nemunaitis JJ, Valone FH, Verjee SS, Jones LA, Hershberg RM. Placebo-controlled phase III trial of immunologic therapy with sipuleucel-T (APC8015) in patients with metastatic, asymptomatic hormone refractory prostate cancer. J Clin Oncol 2006;24(19):3089-3094.

61. Fong L, Brockstedt D, Benike C, Breen JK, Strang G, Ruegg CL, Engleman EG. Dendritic cell-based xenoantigen vaccination for prostate cancer immunotherapy. J Immunol 2001;167(12):7150-7156.

62. Pandha HS, John RJ, Hutchinson J, James N, Whelan M, Corbishley C, Dalgleish AG. Dendritic cell immunotherapy for urological cancers using cryopreserved allogeneic tumour lysate-pulsed cells: a phase I/II study. BJU Int 2004;94(3):412-418.

63. Mu LJ, Kyte JA, Kvalheim G, Aamdal S, Dueland S, Hauser M, Hammerstad H, Waehre H, Raabe N, Gaudernack G. Immunotherapy with allotumour mRNAtransfected dendritic cells in androgen-resistant prostate cancer patients. Br J Cancer 2005;93(7):749-56.

64. Murphy GP, Tjoa BA, Simmons SJ, Rogers MK, Kenny GM, Jarisch J. Higher-dose and less frequent dendritic cell infusions with PSMA peptides in hormone-refractory metastatic prostate cancer patients. Prostate 2000;43(1): 59-62.

65. Noguchi M, Kobayashi K, Suetsugu N, Tomiyasu K, Suekane S, Yamada A, Itoh K, Noda S. Induction of cellular and humoral immune responses to tumor cells and peptides in HLA-A24 positive hormone-refractory prostate cancer patients by peptide vaccination. Prostate 2003; 57(1):80-92.

66. Noguchi M, Itoh K, Suekane S, Yao A, Suetsugu N, Katagiri K, Yamada A, Yamana H, Noda S. Phase I trial of patientoriented vaccination in HLA-A2-positive patients with metastatic hormone-refractory prostate cancer. Cancer Sci 2004;95(1):77-84.

67. Slovin SF, Ragupathi G, Adluri S, Ungers G, Terry K, Kim S, Spassova M, Bornmann WG, Fazzari M, Dantis L, Olkiewicz K, Lloyd KO, et al. Carbohydrate vaccines in cancer: immunogenicity of a fully synthetic globo $\mathrm{H}$ hexasaccharide conjugate in man. Proc Natl Acad Sci U S A 1999;96(10):5710-5715.

68. Slovin SF, Ragupathi G, Musselli C, Olkiewicz K, Verbel D, Kuduk SD, Schwarz JB, Sames D, Danishefsky S, Livingston PO, Scher HI. Fully synthetic carbohydratebased vaccines in biochemically relapsed prostate cancer: clinical trial results with alpha-N-acetylgalactosamine-Oserine/threonine conjugate vaccine. J Clin Oncol 2003;21 (23):4292-4298.

69. Slovin SF, Ragupathi G, Musselli C, Fernandez C, Diani M, Verbel D, Danishefsky S, Livingston P, Scher HI. ThomsenFriedenreich (TF) antigen as a target for prostate cancer vaccine: clinical trial results with TF cluster (c)-KLH plus QS21 conjugate vaccine in patients with biochemically relapsed prostate cancer. Cancer Immunol Immunother 2005;54(7):694-702.

70. North SA, Graham K, Bodnar D, Venner P. A pilot study of the liposomal MUC1 vaccine BLP25 in prostate specific antigen failures after radical prostatectomy. J Urol 2006; 176(1):91-95.

71. Small EJ, Reese DM, Um B, Whisenant S, Dixon SC, Figg WD. Therapy of advanced prostate cancer with granulocyte macrophage colony-stimulating factor. Clin Cancer Res 1999;5(7):1738-1744. 
72. Dreicer R, See WA, Klein EA. Phase II trial of GM-CSF in advanced prostate cancer. Invest New Drugs 2001;19(3): 261-265.

73. Rini BI, Weinberg V, Bok R, Small EJ. Prostate-specific antigen kinetics as a measure of the biologic effect of granulocyte-macrophage colony-stimulating factor in patients with serologic progression of prostate cancer. J Clin Oncol 2003;21(1):99-105.

74. Schwaab T, Tretter CP, Gibson JJ, Cole BF, Schned AR, Harris R, Fisher JL, Crosby N, Stempkowski LM, Heaney JA, Ernstoff MS. Tumor-related immunity in prostate cancer patients treated with human recombinant granulocyte monocyte-colony stimulating factor (GM-CSF). Prostate 2006;66(6):667-674.

75. Pavlenko M, Roos AK, Lundqvist A, Palmborg A, Miller AM, Ozenci V, Bergman B, Egevad L, Hellstrom M, Kiessling R, Masucci G, Wersall P, et al. A phase I trial of DNA vaccination with a plasmid expressing prostate-specific antigen in patients with hormone-refractory prostate cancer. $\mathrm{Br} \mathrm{J}$ Cancer 2004;91(4):688-694.

76. Simons JW, Mikhak B, Chang JF, DeMarzo AM, Carducci MA, Lim M, Weber CE, Baccala AA, Goemann MA, Clift SM, Ando DG, Levitsky HI, et al. Induction of immunity to prostate cancer antigens: results of a clinical trial of vaccination with irradiated autologous prostate tumor cells engineered to secrete granulocyte-macrophage colony-stimulating factor using ex vivo gene transfer. Cancer Res 1999; 59(20):5160-5168.

77. Simons JW, Carducci MA, Mikhak B, Lim M, Biedrzycki B, Borellini F, Clift SM, Hege KM, Ando DG, Piantadosi S, Mulligan R, Nelson WG. Phase I/II trial of an allogeneic cellular immunotherapy in hormone-naive prostate cancer. Clin Cancer Res 2006;12(6):3394-401.

78. Michael A, Ball G, Quatan N, Wushishi F, Russell N, Whelan J, Chakraborty P, Leader D, Whelan M, Pandha H. Delayed disease progression after allogeneic cell vaccination in hormone-resistant prostate cancer and correlation with immunologic variables. Clin Cancer Res 2005;11(12): 4469-4478.

79. Sanda MG, Smith DC, Charles LG, Hwang C, Pienta KJ, Schlom J, Milenic D, Panicali D, Montie JE. Recombinant vaccinia-PSA (PROSTVAC) can induce a prostate-specific immune response in androgen-modulated human prostate cancer. Urology 1999;53:260-6.

80. Eder JP, Kantoff PW, Roper K, Xu GX, Bubley GJ, Boyden J, Gritz L, Mazzara G, Oh WK, Arlen P, Tsang KY, Panicali $\mathrm{D}$, et al. A phase I trial of a recombinant vaccinia virus expressing prostate-specific antigen in advanced prostate cancer. Clin Cancer Res 2000;6(5):1632-1638.

81. Gulley J, Chen AP, Dahut W, Arlen PM, Bastian A, Steinberg SM, Tsang K, Panicali D, Poole D, Schlom J, Michael Hamilton J. Phase I study of a vaccine using recombinant vaccinia virus expressing PSA (rV-PSA) in patients with metastatic androgen-independent prostate cancer. Prostate 2002;53(2):109-117.

82. Kaufman HL, Wang W, Manola J, DiPaola RS, Ko YJ, Sweeney C, Whiteside TL, Schlom J, Wilding G, Weiner LM Phase II randomized study of vaccine treatment of advanced prostate cancer (E7897): a trial of the Eastern Cooperative Oncology Group. J Clin Oncol 2004;22(11): 2122-32.

83. Gulley JL, Arlen PM, Bastian A, Morin S, Marte J, Beetham P, Tsang KY, Yokokawa J, Hodge JW, Menard C, Camphausen K, Coleman $\mathrm{CN}$, et al. Combining a recombinant cancer vaccine with standard definitive radiotherapy in patients with localized prostate cancer. Clin Cancer Res 2005; $11(9): 3353-3362$.
84. Arlen PM, Gulley JL, Parker C, Skarupa L, Pazdur M, Panicali D, Beetham P, Tsang KY, Grosenbach DW, Feldman J, Steinberg SM, Jones E, et al. A randomized phase II study of concurrent docetaxel plus vaccine versus vaccine alone in metastatic androgen-independent prostate cancer. Clin Cancer Res 2006;12:(4)1260-1269.

85. Dipaola R, Plante M, Kaufman H, Petrylak D, Israeli R, Lattime E, Manson K, Schuetz T. A Phase I Trial of Pox PSA vaccines (PROSTVAC(R)-VF) with B7-1, ICAM-1, and LFA3 co-stimulatory molecules (TRICOMtrade mark) in Patients with Prostate Cancer. J Transl Med 2006;4:1.

86. Rosenberg SA, Yang JC, Schwartzentruber DJ, Hwu P, Marincola FM, Topalian SL, Restifo NP, Dudley ME, Schwarz SL, Spiess PJ, Wunderlich JR, Parkhurst MR, et al. Immunologic and therapeutic evaluation of a synthetic peptide vaccine for the treatment of patients with metastatic melanoma. Nat Med 1998;4(3):321-327.

87. Rudge G, Gleeson PA, van Driel IR. Control of immune responses by immunoregulatory T cells. Arch Immunol Ther Exp (Warsz) 2006;54(6):381-391.

88. Beyer M, Schultze JL. Regulatory T cells in cancer. Blood 2006;108(3):804-811.

89. Dannull J, Su Z, Rizzieri D, Yang BK, Coleman D, Yancey D, Zhang A, Dahm P, Chao N, Gilboa E, Vieweg J. Enhancement of vaccine-mediated antitumor immunity in cancer patients after depletion of regulatory T cells. J Clin Invest 2005;115(6):3623-3633.

90. Berd D, Mastrangelo MJ. Effect of low dose cyclophosphamide on the immune system of cancer patients: reduction of T-suppressor function without depletion of the CD8+ subset. Cancer Res 1987;47(12):3317-3321.

91. Ghiringhelli F, Menard C, Puig PE, Ladoire S, Roux S, Martin F, Solary E, Le Cesne A, Zitvogel L, Chauffert B. Metronomic cyclophosphamide regimen selectively depletes $\mathrm{CD} 4(+) \mathrm{CD} 25(+)$ regulatory $\mathrm{T}$ cells and restores $\mathrm{T}$ and NK effector functions in end stage cancer patients. Cancer Immunol Immunother 2007;56(5):641-648.

92. Motoyoshi Y, Kaminoda K, Saitoh O, Hamasaki K, Nakao K, Ishii N, Nagayama Y, Eguchi K. Different mechanisms for anti-tumor effects of low- and high-dose cyclophosphamide. Oncol Rep 2006;16(1):141-146.

93. Dudley ME, Wunderlich JR, Yang JC, Sherry RM, Topalian SL, Restifo NP, Royal RE, Kammula U, White DE, Mavroukakis SA, Rogers LJ, Gracia GJ, et al. Adoptive cell transfer therapy following non-myeloablative but lymphodepleting chemotherapy for the treatment of patients with refractory metastatic melanoma. J Clin Oncol 2005;23(10): 2346-2357.

94. Holtl L, Ramoner R, Zelle-Rieser C, Gander H, Putz T, Papesh C, et al. Allogeneic dendritic cell vaccination against metastatic renal cell carcinoma with or without cyclophosphamide. Cancer Immunol Immunother 2005;54 (7):663-670.

95. Gilboa E. The risk of autoimmunity associated with tumor immunotherapy. Nat Immunol 2001;2(9):789-92.

Correspondencia autor: Dr. H. Veelken

Department of Hematology/Oncology.

University Medical Center Freiburg.

Hugstetter Strasse 55 - D-79106 Freiburg, Germany

Tel.: +49-761-270-7175

E-mail autor: hendrik.veelken@uniklinik-freiburg.de

Información artículo: Original 